\title{
Special Issue on Computer Science Symposium in Russia (2019)
}

\section{René van Bevern ${ }^{1}$. Gregory Kucherov ${ }^{2}$}

Accepted: 14 January 2021 / Published online: 10 February 2021

(C) The Author(s), under exclusive licence to Springer Science+Business Media, LLC part of Springer Nature 2021

This special issue of Theory of Computing Systems consists of a selection of works originally presented at the 14th International Computer Science Symposium in Russia (CSR 2019), held on July 1-5, 2019 in Novosibirsk, Russian Federation. The event was hosted by Novosibirsk State University and chaired by René van Bevern. The conference proceedings appeared in volume 11532 of Springer's Lecture Notes in Computer Science series. The Program Committee of the conference, chaired by Gregory Kucherov, invited authors of several presentations to submit extended versions of their works to this special issue. All papers underwent a rigorous reviewing process following the usual standards of Theory of Computing Systems. As a result, this special issue contains 10 papers out of the 31 papers presented at the conference. The CSR conference series is devoted to a broad range of topics of theoretical computer science, which is reflected in the selected papers. A short description of the 10 contributions presented in this issue follows.

The paper "Derandomization for Sliding Window Algorithms with Strict Correctness" by Moses Ganardi, Danny Hucke, and Markus Lohrey shows that every strictly

This article belongs to the Topical Collection: Special Issue on Computer Science Symposium in Russia (2019)

Guest Editor: René van Bevern and Gregory Kucherov

René van Bevern

rvb@nsu.ru

Gregory Kucherov

gregory.kucherov@univ-mlv.fr

1 Department of Mathematics and Mechanics, Novosibirsk State University, Novosibirsk, Russian Federation

2 CNRS \& Gustave Eiffel University, 77454 Marne-la-Vallée, France 
correct randomized sliding window algorithm can be derandomized without increasing the worst-case space consumption, where a sliding window algorithm is strictly correct if, for every data stream, it outputs, with high probability, a correct value at every time instant.

The paper "On Decidability of Theories of Regular Languages" of Sergey Dudakov and Boris Karlov studies decidability issues for theories of regular languages with the union, concatenation, and Kleene star operations and subsets of those operations. Several new decidability and undecidability results are proved.

The paper "Transition Property for Cube-Free Words" by Elena A. Petrova and Arseny M. Shur contains a series of results about combinatorial properties of cubefree words, both binary and over larger alphabets. In particular, these results answer some open problems raised back in 1985 .

The paper "Nearly Linear Time Isomorphism Algorithms for Some Nonabelian Group Classes" by Bireswar Das and Shivdutt Sharma shows a near linear-time algorithm for testing isomorphism of Hamiltonian groups or of groups that are the direct product of an abelian group and a nonabelian group of bounded order. Herein, it is assumed that the groups are given as multiplication tables with constant-time lookup.

The paper "Parameterized Complexity of Conflict-Free Set Cover" by Ashwin Jacob, Diptapriyo Majumdar, and Venkatesh Raman studies the parameterized complexity of a variant of the Set Cover problem where the sets chosen for the cover must form an independent set in some given graph or matroid.

The paper "Belga B-Trees" by Erik D. Demaine, John Iacono, Grigorios Koumoutsos, and Stefan Langerman belongs to the area of competitive analysis of online algorithms. In relation to the "dynamic optimality conjecture" about binary search trees, the authors study the competitiveness of B-trees under the so-called "diskaccess model". The authors propose a construction of a B-tree, called Belga B-tree, and prove several competitiveness bounds.

The paper "The Non-hardness of Approximating Circuit Size" by Eric Allender, Rahul Ilango, and Neekon Vafa highlights obstacles that would have to be overcome by any proof that the Minimum Circuit Size Problem (MCSP) is hard for NP under $\mathrm{AC}^{0}$ reductions. This may be a step toward confirming a conjecture of Murray and Williams that MCSP is not NP-complete under logtime-uniform $\mathrm{AC}^{0}$ reductions.

The paper "Unpopularity Factor in the Marriage and Roommates Problems" by Suthee Ruangwises and Toshiya Itoh studies the popular matching problem in bipartite and non-bipartite graphs. The study relies on the so-called unpopularity factor of a matching and proposes new algorithms to compute the unpopularity factor for both settings considered.

The paper "Forward Looking Huffman Coding" by Shmuel T. Klein, Shoham Saadia, and Dana Shapira proposes a new approach to dynamic Huffman encoding which is adapted to the input data model. It is shown, in particular, that the method provably outperforms the static Huffman encoding.

The paper "On Tseitin Formulas, Read-Once Branching Programs and Treewidth" by Ludmila Glinskih and Dmitry Itsykson won the best paper award at the conference. It shows that any nondeterministic read-once branching program that decides a 
satisfiable Tseitin formula based on an $n \times n$ grid graph has size at least $2^{\Omega(n)}$. For arbitrary graphs, an exponential lower bound on the branching program size in terms of the treewidth of the graph is given.

In conclusion, we express our deep gratitude to the authors of the papers and to the reviewers for their work. We are also grateful to the Program and Steering Committees of the CSR 2019 conference.

Novosibirsk and Paris, December 2020, R. van Bevern and G. Kucherov.

Publisher's Note Springer Nature remains neutral with regard to jurisdictional claims in published maps and institutional affiliations. 